\title{
High-throughput sequencing of black pepper root transcriptome
}

\author{
Sheila MC Gordo ${ }^{1}$, Daniel G Pinheiro², Edith CO Moreira ${ }^{1}$, Simone M Rodrigues ${ }^{3}$, Marli C Poltronieri ${ }^{3}$, \\ Oriel F de Lemos ${ }^{3}$, Israel Tojal da Silva ${ }^{2}$, Rommel TJ Ramos ${ }^{4}$, Artur Silva ${ }^{4}$, Horacio Schneider ${ }^{1}$, Wilson A Silva Jr ${ }^{2}$, \\ Iracilda Sampaio ${ }^{1}$ and Sylvain Darnet ${ }^{4^{*}}$
}

\begin{abstract}
Background: Black pepper (Piper nigrum L.) is one of the most popular spices in the world. It is used in cooking and the preservation of food and even has medicinal properties. Losses in production from disease are a major limitation in the culture of this crop. The major diseases are root rot and foot rot, which are results of root infection by Fusarium solani and Phytophtora capsici, respectively. Understanding the molecular interaction between the pathogens and the host's root region is important for obtaining resistant cultivars by biotechnological breeding. Genetic and molecular data for this species, though, are limited. In this paper, RNA-Seq technology has been employed, for the first time, to describe the root transcriptome of black pepper.
\end{abstract}

Results: The root transcriptome of black pepper was sequenced by the NGS SOLiD platform and assembled using the multiple-k method. Blast2Go and orthoMCL methods were used to annotate 10338 unigenes. The 4472 predicted proteins showed about 52\% homology with the Arabidopsis proteome. Two root proteomes identified 615 proteins, which seem to define the plant's root pattern. Simple-sequence repeats were identified that may be useful in studies of genetic diversity and may have applications in biotechnology and ecology.

Conclusions: This dataset of 10338 unigenes is crucially important for the biotechnological breeding of black pepper and the ecogenomics of the Magnoliids, a major group of basal angiosperms.

\section{Background}

Black pepper (Piper nigrum) is one of the most popular and oldest spices in the world, with culinary uses and more recently as a food preservative [1]. Due its medicinal properties, it is used in traditional medicine for its antioxidant, anti-inflammatory and anticancer properties $[2,3]$. This species is the second most widely traded spice in the world, accounting for a value of 929 million US dollars from a production of 445900 tons in 2009 [4].

$P$. nigrum is a perennial, climbing vine indigenous to the southwestern region of India. Its production is limited to rainy tropical regions such as those found in Asia and South America. Tropical climates favor the development of diseases, and crop losses due to pests and diseases are a major constraint in the production of black pepper [1]. Resistance against the two major pathogens,

\footnotetext{
* Correspondence: shd@laposte.net

${ }^{4}$ Instituto de Ciências Biológicas, Universidade Federal do Pará, Campus Universitário do Guamá, Rua Augusto Corrêa, n¹, Belém PA 66075-110, Brazil Full list of author information is available at the end of the article
}

Phytophtora capsici and Fusarium solani f. sp. piperis, has not been achieved by classical breeding of the germplasm of cultivated black pepper and remains a major challenge that may be amenable to improvement using plant biotechnology [1]. Despite the agricultural and economic importance of black pepper, knowledge of its genetics is presently very limited [5].

For non-model plants such as black pepper with little or no molecular information available, next-generation sequencing (NGS) technologies offer a great opportunity for the rapid access to genetic information [6-9]. In plant biology, due to large sizes of genomes and high levels of ploidy, the characterization of transcriptomes is a powerful tool for the identification of proteins. RNA-seq can sequence, in an extremely high-throughput and quantitative manner, the transcriptome of an organism or tissue [9]. Nextgeneration sequencing technologies generate large numbers of reads with high sampling rates of cDNA libraries, providing a deeper and more complete view of transcriptomes [8]. This transcriptome-wide description of RNA
C Biomed Central

(c) 2012 Gordo et al.; licensee BioMed Central Ltd. This is an Open Access article distributed under the terms of the Creative Commons Attribution License (http://creativecommons.org/licenses/by/2.0), which permits unrestricted use, distribution, and reproduction in any medium, provided the original work is properly cited. 
sequences identifies the coding sequence, single nucleotide polymorphisms (SNPs), other polymorphisms, splicing variants and relative levels of expression by counting reads, a method known as digital gene expression (DGE) $[9,10]$.

An RNA-seq analysis of the black pepper transcriptome can greatly impact our knowledge of this species. Black pepper belongs to the Piperaceae family of the Magnoliid subclass, know as basal angiosperms, a large and important family for which little genomic data is available (Figure 1). Transcriptomic data could have many ecological and biotechnological repercussions, from exploring and characterizing the evolution of this family, to defining markers such as microsatellites that are useful in breeding programs, to establishing a catalog of expressed genes related to plant defense and metabolism.

In this work, we used RNA-seq technology to analyze the transcriptome of black pepper. About 71 millions reads were generated and 22363 de novo assembled transcripts were obtained from tissue of the stem-root. The transcripts were functionally annotated and represent the first sequences derived from the transcriptome of $P$. nigrum. About 257 new simple sequence repeats (SSRs) were described. These results, the first dataset of sequences of the Magnoliid group, will open new perspectives for studies of diversity and ecology and will benefit breeding and genomic programs aiming to understand plant-pathogen interactions in the root, the organ that is central in the process of infection by F. solani and P. capsici, the two major pathogens of black pepper.

\section{Results}

\section{Sequencing results}

The dataset of raw reads was deposited in NCBI database under SRA047721 accession number and contains about 71878419 reads $50 \mathrm{bp}$ in length totalling $3.5 \mathrm{Gbp}$ (Table 1). Pre-processing was performed with high stringency; reads with quality values $(\mathrm{QVs})$ below 20 in windows averaging $3 \mathrm{bp}$ and bases with QVs below 18 were trimmed. Reads shorter than $30 \mathrm{bp}$ after pre-processing were excluded. After removing redundant reads, filtering reads of low quality, trimming low-quality ends and removing adapters, the size of the dataset was 13300000 millions reads, representing about $665 \mathrm{Mbp}$. Additional file 1: Figure S1 shows the QVs of the filtered read dataset as monitored by read position using FASTQC [11]. The average QV varies from 29 to 22 (base call accuracy of 99.37-99.85\%), with a slow decrease along the read length. No bases with QVs under eight (base call accuracy under $85 \%$ ) were observed, and more than $75 \%$ of bases had a QV over 12 (base call accuracy over 93.7\%). Additional file 2: Figure S2 presents the length distribution of reads; $83 \%$ of reads have a length over $45 \mathrm{bp}$ after trimming and filtering.

\section{De novo transcriptome assembly}

The assembly of the transcriptome from the stem-root of black pepper was performed using the method of additive multiple-k described by Surget-Groba et al. [12]. The first step of this method is to assemble the reads using single k-mers varying from 19 to 43 bases. Figure 2 presents the results of assembly for each k-mer. The numbers of contigs decrease from 6847 ( $\mathrm{k}-\mathrm{mer}=19$ bases) to 22 ( $\mathrm{k}$-mer $=43$ bases). The N50 and average length of contigs inversely increase from $166 \mathrm{bp}$ to $214 \mathrm{bp}$ and $151 \mathrm{bp}$ to $236 \mathrm{bp}$, respectively, from k-mers of 19 to 43 bases in length (Figure 2). Thus, the average length of contigs is greater from larger k-mers, but the number of contigs generated is lower. The second step

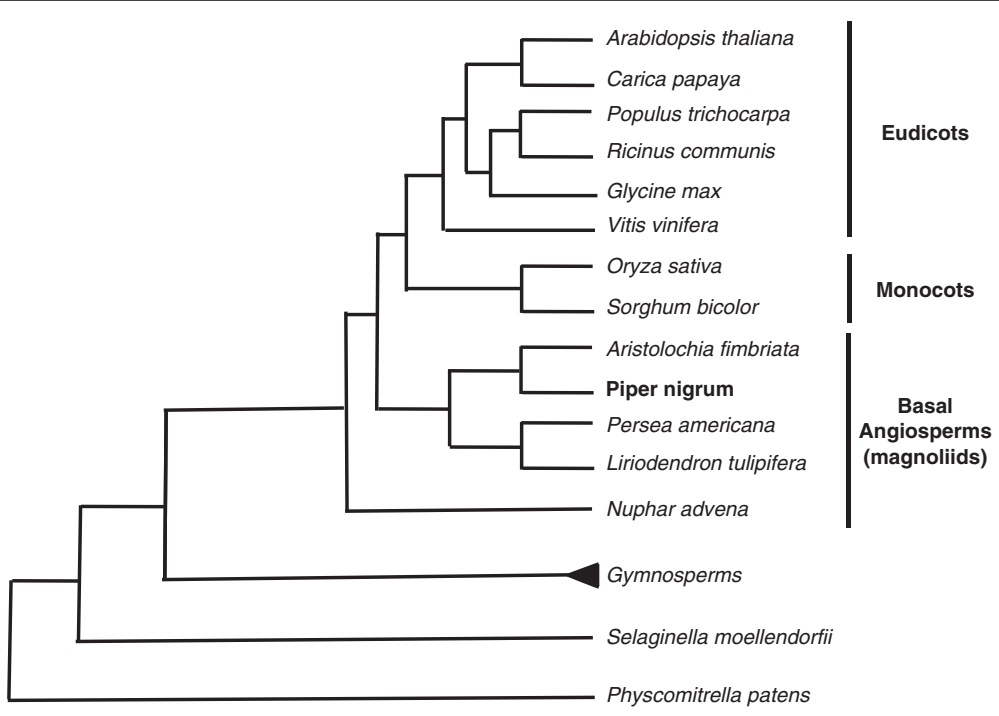

Figure 1 Phylogenetic position of Piper nigrum with others plants group. 
Table 1 Overview of sequencing and assembly

\begin{tabular}{ll}
\hline Raw data & \\
\hline Total number of reads & 71878419 \\
Number of unique high-quality reads & 13300000 \\
Number of contigs obtained with Velvet/Oases/STM & 22363 \\
Unigenes dataset & \\
Unigenes number & 10338 \\
Total size (bp) & 1787600 \\
Largest transcript length (bp) & 1314 \\
N50 length (bp) & 168 \\
No ATGC characters & 18 \\
Average coverage per base & 62 \\
Predicted proteins & \\
Predicted CDS (partial/complete) & 4472 \\
Gene expression & 71148200 \\
Reads mapped in transcripts & 68250 \\
\hline
\end{tabular}

bp: base pair; rpkm: read coverage normalized per million mapped nucleotides and the length of unigene.

of the additive multiple-k method is to merge the assemblies using different k-mers, removing redundant contigs. This method has the advantage to detect low expressed genes and improve the assembly of long contigs. To identify possible isoforms of transcripts, the assembly was performed using the Oases script, which clusters similar contigs into small groups to derive isoforms of transcripts. The $\mathrm{STM}^{-}$methods was used, considering the Aristolochia fimbriata predicted proteome as reference to generate scaffolds.

A final dataset of 22363 contigs was obtained, corresponding to $3.8 \mathrm{Mbp}$. After contigs assembly, the contigs dataset was filtered using SeqClean tool and were removed 1357 contigs with rDNA sequences, 1640 with mitochondrial, 539 with chloroplastidial and 154 plant DNA repeats sequences (http://seqclean.sourceforge.net/). All contigs with a length inferior to $100 \mathrm{bp}$ were excluded.

Following the cleanup step, iAssembler tool was used to cluster and assembly contigs to obtain unigene sequences [13]. Finally, 10338 unigenes were identified from contigs dataset. The largest unigene is $1314 \mathrm{bp}$ in length, and the N50 length is $168 \mathrm{bp}$ (Table 1, Figure 3).

The average read coverage is 211 reads per contig, and the highest coverage is 46239 reads for one contig, corresponding to $68250 \mathrm{in} \mathrm{rpkm}$ unit. The average coverage per base is $62 x$, which indicates that, on average, each base of the contigs was sequenced 62 -fold. For example, the base coverage of contig NODE-882-2-0_930, one the longest of the dataset, was plotted, and the coverage per base varied from one to 22 along the 959 bp (Figure 4).

\section{Functional annotation of the transcriptome}

The number of predicted partial or complete coding sequences (CDS) was 4472, representing about $44 \%$ of the total transcripts. An ncRNA search of the NOCODE databank [14] indicated that only 32 reads had significant homology with non-coding RNAs, with putative regulatory functions. About $52 \%$ of predicted proteins had a significant homology (limit of $1 \mathrm{e}^{-05}$ ) with Arabidopsis thaliana proteins sequences in the NCBI database (Table 2). The species with the highest hit rates were Populus trichocarpa with 54.38\%, Aristolochia fimbriata with $54.02 \%$ and Vitis vinifera with $53.93 \%$ of hits. The percentage of sequences from our dataset showing homology $\left(1 \mathrm{e}^{-5}\right)$ with proteomes of five model plants is reported in Table 2. The homology varied from $50 \%$ to $54 \%$, with lower values obtained with monocots than with dicots. In Figure 4, the contig NODE-882-2-0_930 was aligned to the mRNA sequence of the putative premRNA-splicing factor, RBM22/SLT11, from Populus trichocarpa. The P. nigrum contig and the P. trichocarpa mRNA show $77 \%$ identity in nucleotides along the

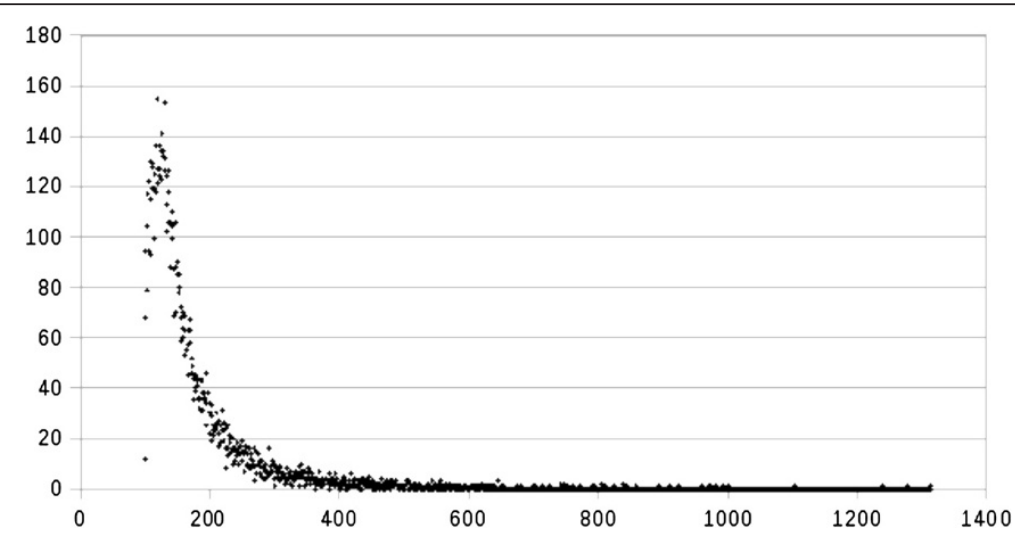

Figure 2 Comparison of de novo assembly for different k-mers. The bars indicate the number of contigs generated, and the hashed portions represent the percent of contigs that have homology in the databank of non-redundant proteins using BlastX. The lines indicate the N50 length (triangle) and the average length (lozenge) in bp. X-axis: k-mer number; Y-axis: read count. 


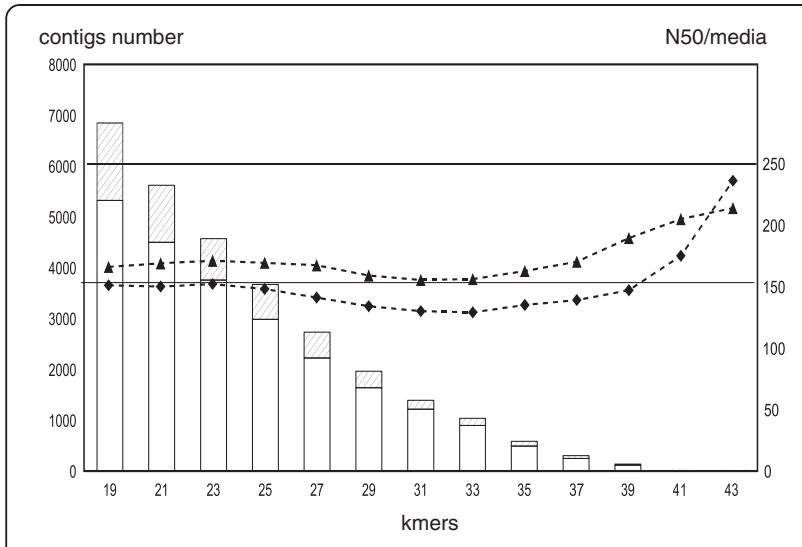

Figure 3 Size distribution of the transcripts from 2-GS sequencing from the black pepper root. Y-axis: count number; X-axis: size in bp.

putative CDS sequence. The corresponding protein sequences have an identity of $87 \%$ and a similarity of $96 \%$, with a coverage of $94 \%$, without gaps.

The functional annotation was performed using BLAST2GO results [15], and 3055 (29.5\% of total unigenes) were functionally annotated with the gene ontology (GO) databank for P. nigrum root library and 9664 unigenes $(73.3 \%)$ for one $A$. thaliana root library (AtGI5336) used for comparison. The GO-SLIM annotations for black pepper root and one Arabidopsis thaliana root library are graphically presented in Figure 5 . The biological processes with higher numbers of transcripts are in the categories "cellular process" and "metabolic process", with respectively 1499 and 1328 unigenes. The "Molecular Function" classification reveals that the function "binding" is at a similar level as "catalytic activity", with respectively 1712 and 1772 unigenes. The "cell part" annotations, as shown in Figure 5, indicate that a

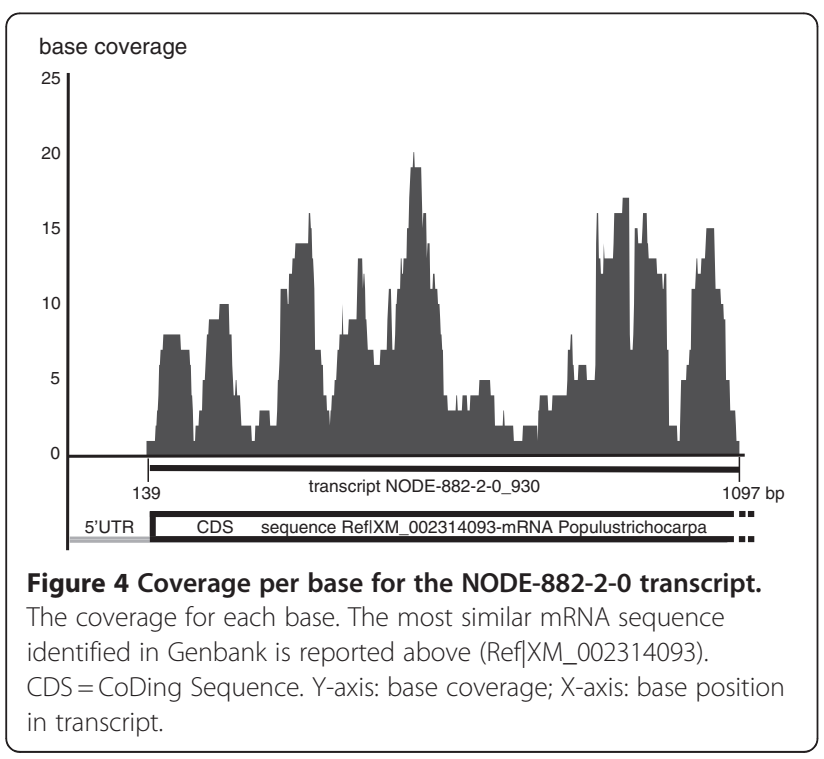

high proportion, about $39 \%$ of predicted CDS, should be localized in organelles. In Table 3 was reported the proteins number for the $A$. thaliana and $P$. nigrum root. Using the bioinformatics tools was predicted from cDNA sequences, 8004 protein sequences for A. thaliana root and 4472 for $P$. nigrum (Table 3 ). Using orthoMCL tool, were identified 3408 orthogroups and 37 paralogs for A. thaliana and 1017 and 159 for $P$. nigrum. The proportion of orthogroups/proteins number for each plant is different, $42.6 \%$ for $A$. thaliana and $22.7 \%$ for $P$. nigrum, and should be explained by the fact that $A$. thaliana is diploid and $P$. nigrum is tetraploid.

\section{Detection of microsatellites}

Microsatellites were identified using the MISA script (http://pgrc.ipk-gatersleben.de/misa/). Analyzing the 22363 contigs, 168 di-, tri- and tetranucleotide repeats were identified, representing $43 \%, 56 \%$ and $1 \%$ of the total, respectively (Table 4). The most commonly detected SSR was the trinucleotide AGC, with ACT and ACG the second most common.

\section{Discussion}

Biotechnological methods are being successfully employed in plant breeding [16] to confer, for example, increased resistances to pathogens. To use biotechnology in breeding programs, however, prior genetic and genomic knowledge is needed to isolate genes and characterize genotypes and phenotypes [16]. Plant genomics, especially for non-model plants, is always a challenge due to the high levels of ploidy, large genome sizes, low genome complexities and large proportions of repeat sequences. Instead of sequencing the complete genome, sequencing the transcriptome is a good alternative for rapidly and efficiently accessing the expressed genes and for characterizing phenotypes [17].

The characterization of transcriptomes can use hybrid- or sequenced-based technologies, but all hybrid-based technologies require prior knowledge of the transcriptome. In plant biology, Expressed Sequence

Table 2 Percent of predicted proteins with homology to protein sequences from databases of other species

\begin{tabular}{ll}
\hline Protein databases & \% with homology \\
\hline Arabidopsis thaliana & 51.57 \\
Glycine max & 53.23 \\
Vitis vinifera & 53.93 \\
Oryza sativa & 50.87 \\
Populus trichocarpa & 54.38 \\
Sorghum bicolor & 51.43 \\
Aristolochia fimbrata & 54.02 \\
\hline
\end{tabular}

Predicted protein sequences were obtained used FrameD tool and BLASTP was performed with e-value of $1 \mathrm{e}^{-05}$. 


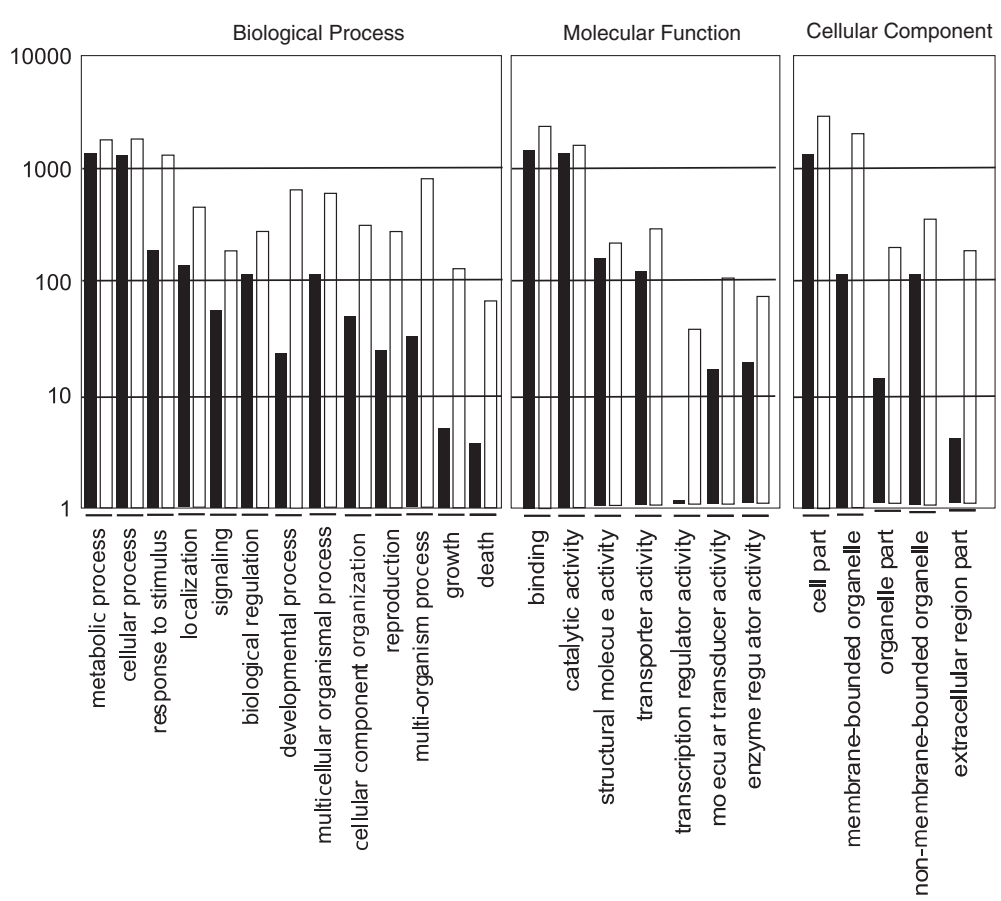

Figure 5 Gene ontology classification of transcripts. Black bar: P. nigrum root library; White bar: A. thaliana root library.

Tags (ESTs) based on the sequencing of cDNA libraries have been a great advance and have contributed significantly to the study of plant genomics. The sequencing of cDNA libraries, however, has many limitations, such as the cost in time and money involved in cloning and Sanger sequencing. The serial analysis of gene expression (SAGE) and the improved variant SuperSAGE have been used to increase throughput and sampling, but the reliability of the identification of transcripts is one of their limitations [18]. The second generation of sequencing (2-GS) technology circumvents these limitations and generates a large number of reads or datasets of short reads in a shorter time and with a lower

Table 3 Characterization of proteome predicted from CDS of root libraries

\begin{tabular}{lll}
\hline & \multicolumn{2}{l}{ Proteins number } \\
\hline Arabidopsis thaliana root library 5536 & 8004 & \\
Black pepper root (our work) & 4472 & \\
& OrthoMCL proteins families \\
Arabidopsis thaliana root library 5536 & Orthogroups & 3408 \\
& Paralogs & 37 \\
& No group & 137 \\
Black pepper root (our work) & Orthogroups & 1017 \\
& Paralogs & 159 \\
& No group & 68 \\
\hline
\end{tabular}

reagent cost per base than Sanger sequencing. 2-GS technologies have opened great prospectives for genetic studies of non-model plants [17].

\section{Table 4 SSR statistics}

\begin{tabular}{lll}
\hline Dinucleotide repeat & number & $\%$ \\
\hline AC & 21 & 29 \\
AG & 44 & 60 \\
AT & 5 & 7 \\
CG & 3 & 4 \\
Total & 73 & 100 \\
Trinucleotide repeat & number & $\%$ \\
AAC & 7 & 7 \\
AAG & 7 & 7 \\
AAT & 8 & 9 \\
ACC & 1 & 1 \\
ACG & 15 & 16 \\
ACT & 15 & 16 \\
AGC & 18 & 19 \\
AGG & 6 & 6 \\
AGT & 10 & 10 \\
CCG & 7 & 7 \\
Total & 94 & 100 \\
Tetranucleotide repeat & number & \\
AAAT/ATT & 1 & \\
\hline
\end{tabular}


The genome of black pepper is very poorly characterized, with only 184 sequences deposited in Genbank (access 3/11/2011). Previous genetic studies have focused on the diversity, phylogeny and taxonomy of cultivated black pepper and its wild relatives [19-22]. Despite the agricultural and economic importance of this spice, only DNA sequences of phylogenetics markers are available in molecular databases (Figure 1). Another difficulty for the genomic study of black pepper is the lack of a sequenced genome from the Magnoliid group, describe as basal angiosperms group [23,24] (Figure 1). All fullsequenced genomes available in databases are from monocot or dicot plants, plants phylogenetically distant from black pepper, as show in Figure $1[23,24]$.

About $20 \mathrm{~Gb}$ of the data can be mapped, corresponding to 71 million short reads of $50 \mathrm{bp}$. De novo assembly generated 22363 contigs, 10338 unigenes with an N50 of $168 \mathrm{bp}$. The N50 is slightly less than the $202 \mathrm{bp}$ obtained in an Illumina $75 \mathrm{bp}$ dataset for the Ipomoea batatas transcriptome and the $208 \mathrm{bp}$ obtained in a $75 \mathrm{bp}$ single- and paired-end dataset from Camellia sinensis $[25,26]$. The number of contigs, however, is lower than in these other studies: 56516 and 127094 contigs for $I$. batatas and C. sinensis, respectively. Although that the read number is higher in our study, (71 million vs 59 and 35 million for I. batatas and C. sinensis), the contig number is inferior. This result could be explain by the read lengths were different (50 bp vs $75 \mathrm{pb}$ ) and SOLiD dataset is lower in quality, only $50-60 \%$ of reads mapped against a transcriptome reference $[27,28]$.

Estimating the amount of coverage of the transcriptome is complex due to the lack of genetic data from black pepper. Black pepper is tetraploid with $2 n=52$. The size of its genome, estimated by cytogenetic studies, is about $1220 \mathrm{Mbp}(1 \mathrm{C}=1.25 \mathrm{pg})$, which is about tenfold larger than the genome of Arabidopsis thaliana [29]. In $A$. thaliana, about $6 \%$ of the genome is transcribed, representing 41671 transcripts [18]. In the $A$. thaliana TGI database, however, two root-derived cDNA libraries have largely been sequenced and have identified 5609 and 5884 transcripts (11.3 and $9.3 \mathrm{Mbp}$ ), which seems to indicate that only $14 \%$ of predicted genes are expressed in the root [30]. Assuming a similar proportion of transcription in $P$. nigrum, the entire transcriptome is estimated to be $73.2 \mathrm{Mbp}(6 \%)$ and the root transcriptome to be $10.3 \mathrm{Mbp}(0.84 \%)$.

The cDNA library was sequenced with a high coverage per base, $62 \mathrm{x}$ on average, summing $3.8 \mathrm{Mbp}$. Shown as an example in Figure 4, the transcript NODE-882-2-0_930 has a good read coverage, up to 20x, and shows a high identity and a good co-linearity with an mRNA sequence from $P$. trichocarpa, indicating a good quality of sequencing and assembly. The comparison of predicted CDSs with the proteomes of other plants demonstrates that the percentage of sequences with significant homology is higher with dicots $(51-53 \%)$ than with monocots (15$23.4 \%)$. This low degree of homology may be due to the phylogenetic distance between magnoliids and others groups (eucots and dicots) and to the different average transcript size $(211 \mathrm{pb})$, as described during the sequencing of the I. batatas transcriptome [25]. The method of k-mer additive assembly is efficient in maximizing the number of transcripts, and interestingly, the proportion of transcripts with significant homology (22-35\%), the N50 (129 to $236 \mathrm{bp}$ ) and the average length of transcript (156$214 \mathrm{bp}$ ) are relatively well conserved for each k-mer (19$43 \mathrm{bp)}$ (Figure 2). Comparison with other studies of plant transcriptomes is difficult because the read length used with the Illumina platform is longer (75 bp) and the library is paired-end, which clearly facilitates the process of assembly, as demonstrated for I. batatas [25]. Finally, limitations of the 2-GS platform produce high numbers of short contigs, but our dataset has 2144 unigenes over $200 \mathrm{bp}$ in length and about $72 \%$ of reads with a significant homology, which represent a significant advance in our biological knowledge of black pepper.

The functional annotation of the dataset of transcripts is informative for the physiology of stems and roots of black pepper. NGS datasets from plant root tissue are only available for ginseng and sweetpotato. The "response to stimulus" and "localization" categories of Figure 5 are highly represented in the transcriptomes from roots of both sweetpotato and black pepper [25]. The profile of molecular functions is conserved between the two root plants. In both, the function "transporter activity" is quite high. This feature may be explained by the fact that roots are very important in the absorption of microelements. This preliminary annotation of the transcriptome from black pepper is very important and should lead to the identification of new genes coding for transporters, transcription factors specific to root and stem tissue or proteins used for defence.

\section{Conclusion}

In model plants, NGS has increased the ability to generate and analyze the expression and detection of most transcripts, to identify genes and their forms of alternative splicing and to analyze DNA methylation and histone modifications on a genomic scale $[6,8]$. The technology based on Sanger sequencing has generated much data from model species, and plants with sequenced genomes and transcriptomes have provided a robust database reflecting gene expression. The transcriptomic resources for non-model plants, particularly with NGS, however, are limited, especially for the analysis of expressed sequences, alternative splicing and SNPs.

The sequencing of $P$. nigrum using the SOLiD platform (short-reads) produced the first dataset of sequences for a non- 
model species belonging to the Magnoliid group and order Piperales. Assembly without a reference genome or transcriptome is considered difficult, but a partial transcriptome of the non-model species $P$. nigrum was assembled. This work was aided by the use of some de novo assemblies performed using NGS datafrom non-model plants such as chickpea Cicer arietinum L. [31], Eucalyptus grandis x Eucalyptus urophylla hybrid [32],rubber Hevea brasiliensis [33], sweetpotatoIpomoeabatatas [25] andbuckwheat Fagopyrum [34].

Analysis of SSRs in the data generated has great potential for applications in studies of genetic diversity, plant breeding and reproduction. The sequences produced in this study are very relevant, sincelittle information aboutblack pepper is available in thebiological databases, and the data produced can be used in ecological studies andbiotechnology.

\section{Methods}

Plant material

The tissue samples were obtained from basal root region of eight 70-day-old plantlets. All plantlets used in this study are from Brazilian cultivar of P. nigrum, Bragantina (Additional file 3: Figure S3). The Bragantina cultivar is an ecotype of the Asian Panniyur-1 cultivar, which is a hybrid of the Uthirankotta and Cheriyakaniyakadan cultivars. Stakes obtained fromblackpepper plants were rooted in trays containing sand and grown in pots containing vermiculite previously autoclaved twice. The plantlets used in this study were grown and acclimatized in a greenhouseforaboutaround two months.

\section{cDNA preparation and sequencing}

Total RNA was extracted from $35 \mathrm{mg}$ of tissue from the stemroot region of $P$. nigrum using the Illustra RNAspin Mini Kit (GE Healthcare, USA). To remove any DNA contamination, the sample was cleaned with the Oligotex Direct mRNA Mini Kit(Quiagen,USA). TherRNAs were depleted using a RiboMinus Eukaryote Kit for RNA-Seq and a RiboMinus Plant Kit for RNA-Seq (Invitrogen, USA). The concentration of the mRNA wasdeterminedusinga Qubitanalyzer(Invitrogen,USA).

An aliquote $(126.5 \mathrm{ng} / \mu \mathrm{L})$ of depleted RNA was fragmented and used to generate one cDNA library for high-throughputsequencing. The library of fragments was obtained using the SOLiD Total RNA-Seq Kit (Invitrogen, USA). The global procedure of this kit is based on the hybridization of adapters with degenerate ends, followed by reverse transcription and library amplification by PCR [35]. The cDNAs were selected by size on a polyacrylamide gel before and after the library amplification. The sequencing of the cDNA library was performed in a well of aflow cell.

\section{Pre-processing sequencing data}

The SOLiD adapter sequences were trimmed from reads using CutAdapt (0.9.5) [36], and the sequences of reads were converted from SOLiD encoding color space into double-encoded colors in FASTQ format. PRINSEQ (lite
0.14.4) [37] was used to remove redundant reads, to trim low quality reads (average $\mathrm{QV}<20$ for a 3 -nt window, and $\mathrm{QV}<18$ ) and to remove small pre-processed reads (read length $<30 \mathrm{bp}$ ) [37]. Statistics of the dataset of reads was obtained using FastQC software (V. 0.9.2) (http://www.bioinformatics.bbsrc.ac.uk/projects/fastqc/).

\section{De novo transcriptome assembly and functional annotation}

Transcriptome assembly was performed using Velvet and Oases [38,39]. For optimizing the de novo assembly, the method of additive multiple-k [12] was used to combine the properties of multiple assemblies using different k-mers (19-43).

The program ESTScan was used to detect potential coding regions in the transcript sequences obtained by assembly [40]. In order to obtain the protein sequence the FrameD tool was employed [41]. The program BWA [42] was used to align raw reads against all contigs obtained by Velvet assembly for calculating the coverage of the sequencing.

Local BLASTX (E-value $1 \mathrm{e}^{-05}$ ) was used to search homologous sequences against plant databases from PlantGDB (ftp://ftp.plangdb.org), nr-viridiplantae (NCBI), $V$. vinifera, A. thaliana, P. trichocarpa, G. max and O. sativa.

The functional annotation was based on BLASTX results with the nr-viridiplantae database, and the program BLAST2GO was used to assign biological functions, cellular components and cellular processes to the transcripts [43]. The groups of orthologous and paralogous proteins were predicted using orthoMCL tools [44].

\section{Detection of microsatellites}

The MISA perl script was used to identify the position of SSRs in the sequence dataset (http://pgrc.ipk-gatersleben.de/misa/misa.html). The search for SSRs in our transcriptome was performed using the detection of bi-, tri- and tetranucleotide repeats, present more than $5 \mathrm{x}$ and with a minimum distance of $100 \mathrm{bp}$.

\section{Additional files}

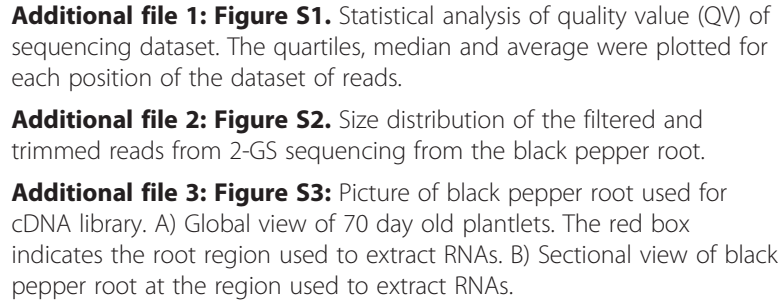

Additional file 1: Figure S1. Statistical analysis of quality value (QV) of sequencing dataset. The quartiles, median and average were plotted for each position of the dataset of reads.

Additional file 2: Figure S2. Size distribution of the filtered and trimmed reads from 2-GS sequencing from the black pepper root. Additional file 3: Figure S3: Picture of black pepper root used for cDNA library. A) Global view of 70 day old plantlets. The red box indicates the root region used to extract RNAs. B) Sectional view of black pepper root at the region used to extract RNAs.

Competing interests

The authors declare that they have no competing interests. 


\section{Authors' contributions}

Conceived and designed the experiments: SMCG, ECOM, SMR, AS, HS, WASJr, MICS, SD. Performed the experiments: SMCG, DGP, ECOM, SMR, MCP, ORFL, IT, RTJR, SD. Contributed reagents/materials/analysis tools: SMR, AS, HS, WASJr, MICS. Wrote the paper: SMCG, AS, HS, WASJr, MICS, SD. All authors read and approved the final manuscript.

\section{Acknowledgments}

The authors thank, MSR. Barbosa for technical help. The work was supported by the Rede Paraense de Genômica e Proteômica (Governo do Estado do Pará/SEDECT/FAPESPA), PROPESP/UFPA, FADESP, FINEP and CAPES (Coordenação de Aperfeicoamento de Pessoal de Nivel Superior).

\section{Author details}

${ }^{1}$ Genetics and Molecular Biology Laboratory, Coastal Studies Institute, Bragança Campus, Universidade Federal do Pará, Bragança, PA 68.600-000, Brazil. ²Departamento de Genética, Faculdade de Medicina de Ribeirão Preto, Universidade de São Paulo, Centro Regional de Hemoterapia de Ribeirão Preto, Rua Tenente Catão Roxo, 2501, Ribeirão Preto SP 14051-140, Brazil. ${ }^{3}$ EMBRAPA Amazônia Oriental, Trav. Dr. Enéas Pinheiro s/nº, Caixa Postal 48, Belém PA 66095-100, Brazil. ${ }^{4}$ Instituto de Ciências Biológicas, Universidade Federal do Pará, Campus Universitário do Guamá, Rua Augusto Corrêa, no1, Belém PA 66075-110, Brazil.

Received: 26 January 2012 Accepted: 29 August 2012

Published: 17 September 2012

\section{References}

1. Nair KPP: Agronomy and Economy of Black Pepper and Cardamom The "King" and "Queen" of Spices. New York: Elsevier; 2011.

2. Nishimura Y, Kitagishi Y, Yoshida H, Okumura N, Matsuda S: Ethanol extracts of black pepper or turmeric down-regulated SIRT1 protein expression in Daudi culture cells. Mol Med Report 2011, 4:727-730.

3. Liu Y, Yadev VR, Aggarwal BB, Nair MG: Inhibitory effects of black pepper (Piper nigrum) extracts and compounds on human tumor cell proliferation, cyclooxygenase enzymes, lipid peroxidation and nuclear transcription factor-kappa-B. Nat Prod Commun 2010, 5:1253-1257.

4. Food and Agriculture Organization of the United Nations: Statistics of Agricultural Production. Rome: FAO; 2011.

5. Dias JS, Ortiz R: Transgenic Vegetable Crops: Progress, Potentials, and Prospects. In Plant Breeding Reviews, Plant Breeding Reviews, Volume 35. Edited by Janick J. New York: Wiley Blackwell; 2011:151-224. Janick J. (Series Editor).

6. Hawkins RD, Hon GC, Ren B: Next-generation genomics: an integrative approach. Nat Rev Genet 2010, 11(7):476-486.

7. Lister R, Gregory BD, Ecker JR: Next is now: new technologies for sequencing of genomes, transcriptomes, and beyond. Curr Opin Plant Biol 2009, 12(2):107-118

8. Morozova O, Hirst M, Marra MA: Applications of new sequencing technologies for transcriptome analysis. Annu Rev Genomics Hum Genet 2009, 10:135-151.

9. Wang Z, Gerstein M, Snyder M: RNA-Seq: a revolutionary tool for transcriptomics. Nat Rev Genet 2009, 10(1):57-63.

10. Nagalakshmi U, Waern K, Snyder M: RNA-Seq: a method for comprehensive transcriptome analysis. Curr Protoc Mol Biol 2010, SUPPL. 89:4.11.1-4.11.13.

11. Babraham Bioinformatics: FASTQC, a quality control tool for the high throughput sequence data.; 2012 http://www.bioinformatics.bbsrc.ac.uk/ projects/fastqc/.

12. Surget-Groba Y, Montoya-Burgos Jl: Optimization of de novo transcriptome assembly from next-generation sequencing data. Genome Res 2010, 20:1432-1440.

13. Zheng $Y$, Zhao $L$, Gao J, Fei Z: iAssembler: a package for de novo assembly of Roche-454/Sanger transcriptome sequences. BMC Bioinformatics 2011, 12:453.

14. He S, Liu C, Skogerbø G, Zhao H, Wang J, Liu T, Bai B, Zhao Y, Chen R: NONCODE v2.0: decoding the non-coding. Nucleic Acids Res 2008, 36 D170-D172.

15. Gotz S, Garcia-Gomez JM, Terol J, Williams TD, Nagaraj SH, Nueda MJ, Robles M, Talon M, Dopazo J, Conesa A: High-throughput functional annotation and data mining with the Blast $2 \mathrm{GO}$ suite. Nucleic Acids Res 2008, 36(10):3420-3435.

16. Langridge P, Fleury D: Making the most of 'omics' for crop breeding. Trends Biotechnol 2011, 29(1):33-40.

17. Wang L, Li P, Brutnell TP: Exploring plant transcriptomes using ultra high-throughput sequencing. Brief Funct Genomic Proteomic 2010, 9(2):118-128.

18. Gong W, Shen YP, Ma LG, Pan Y, Du YL, Wang DH, Yang JY, Hu LD, Liu XF, Dong CX, et al: Genome-wide ORFeome cloning and analysis of Arabidopsis transcription factor genes. Plant Physiol (Rockv) 2004, 135(2):773-782.

19. Jaramillo MA, Manos PS: Phylogeny and patterns of floral diversity in the genus Piper (Piperaceae). Am J Bot 2001, 88(4):706-716.

20. Menezes IC, Cidade FW, Souza AP, Sampaio IC: Isolation and characterization of microsatellite loci in the black pepper, Piper nigrum L. (piperaceae). Conserv Genet Resour 2009, 1(1):209-212.

21. Sen S, Skaria R, Abdul Muneer PM: Genetic diversity analysis in Piper species (Piperaceae) using RAPD markers. Mol Biotechnol 2010, 46(1):72-79.

22. Joy $\mathrm{N}$, Abraham Z, Soniya EV: A preliminary assessment of genetic relationships among agronomically important cultivars of black pepper. BMC Genet 2007, 8:42.

23. Paterson AH: Leafing through the genomes of our major crop plants: strategies for capturing unique information. Nat Rev Genet 2006, 7(3):174-184.

24. Jiao Y, Wickett NJ, Ayyampalayam S, Chanderbali AS, Landherr L, Ralph PE, Tomsho LP, Hu Y, Liang H, Soltis PS, et al: Ancestral polyploidy in seed plants and angiosperms. Nature 2011, 473(7345):97-100.

25. Wang Z, Fang B, Chen J, Zhang X, Luo Z, Huang L, Chen X, Li Y: De novo assembly and characterization of root transcriptome using Illumina paired-end sequencing and development of cSSR markers in sweet potato (Ipomoea batatas). BMC Genomics 2010, 11:726

26. Shi CY, Yang H, Wei CL, Yu O, Zhang ZZ, Jiang CJ, Sun J, Li YY, Chen Q, Xia $T$, et al: Deep sequencing of the Camellia sinensis transcriptome revealed candidate genes for major metabolic pathways of tea-specific compounds. BMC Genomics 2011, 12:131.

27. Goff L, Davila J, Swerdel M, Moore J, Cohen R, Wu H, Sun Y, Hart R: Ago2 immunoprecipitation identifies predicted microRNAs in human embryonic stem cells and neural precursors. PLoS One 2009, 4(9):e7192.

28. Ribeiro-dos-Santos A, Khayat AS, Silva A, Alencar DO, Lobato J, Luz L, Pinheiro DG, Varuzza L, Assumpcao M, Assumpcao P, et al: Ultra-deep sequencing reveals the microRNA expression pattern of the human stomach. PLoS One 2010, 5(10):e13205.

29. Soltis DE, Soltis PS, Bennett MD, Leitch LJ: Evolution of genome size in the angiosperms. Am J Bot 2003, 90:1596-1603.

30. Quackenbush J, Cho J, Lee D, Liang F, Holt I, Karamycheva S, Parvizi B, Pertea G, Sultana R, White J: The TIGR Gene Indices: analysis of gene transcript sequences in highly sampled eukaryotic species. Nucleic Acids Res 2001, 29(1):159-164.

31. Garg R, Patel RK, Jhanwar S, Priya P, Bhattacharjee A, Yadav G, Bhatia S, Chattopadhyay D, Tyagi AK, Jain M: Gene Discovery and Tissue-Specific Transcriptome Analysis in Chickpea with Massively Parallel Pyrosequencing and Web Resource Development. Plant Physiol (Rockv) 2011, 156:1661-1678.

32. Mizrachi E, Hefer CA, Ranik M, Joubert F, Myburg AA: De novo assembled expressed gene catalog of a fast-growing Eucalyptus tree produced by Illumina mRNA-Seq. BMC Genomics 2010, 11:681.

33. Xia Z, Xu H, Zhai J, Li D, Luo H, He C, Huang X: RNA-Seq analysis and de novo transcriptome assembly of Hevea brasiliensis. Plant Mol Biol 2011, 77(3):299-308

34. Logacheva MD, Kasianov AS, Vinogradov DV, Samigullin TH, Gelfand MS, Makeev VJ, Penin AA: De novo sequencing and characterization of floral transcriptome in two species of buckwheat (Fagopyrum). BMC Genomics 2011, 12:30

35. Applied Biosystems: SOLiD ${ }^{\mathrm{TM}}$ Total RNA-Sea Kit. 2010.

36. Martins $M$ : Cutadapt removes adapter sequences from high-throughput sequencing reads. EMBnet.journal 2011, 17(1):10-12.

37. Schmieder R, Edwards R: Quality control and preprocessing of metagenomic datasets. Bioinformatics 2011, 27:863-864.

38. Schulz MH, Zerbino DR, Vingron MA, Birney EC: Oases: Robust de novo RNA-seq assembly across the dynamic range of expression levels. Bioinformatics 2012, 28(8):1086-1092.

39. Zerbino DR, Birney E: Velvet: algorithms for de novo short read assembly using de Bruijn graphs. Genome Res 2008, 18:821-829. 
40. Iseli C, Jongeneel CV, Bucher P: ESTScan: a program for detecting, evaluating, and reconstructing potential coding regions in EST sequences. Proc Int Conf Intell Syst Mol Biol 1999, ISMB99:138-148

41. Schiex T, Gouzy J, Moisan A, de Oliveira Y: FrameD: A flexible program for quality check and gene prediction in prokaryotic genomes and noisy matured eukaryotic sequences. Nucleic Acids Res 2003, 31(13):3738-3741.

42. Li H, Durbin R: Fast and accurate short read alignment with BurrowsWheeler transform. Bioinformatics 2009, 25(14):1754-1760.

43. Conesa A, Gotz S: Blast2GO: A comprehensive suite for functional analysis in plant genomics. Int J Plant Genomics 2008, 2008:619832

44. Fischer S, Brunk BP, Chen F, Gao X, Harb OS, lodice JB, Shanmugam D, Roos DS, Stoeckert CJ: Using OrthoMCL to assign proteins to OrthoMCL-DB groups or to cluster proteomes into new ortholog groups. Curr Protoc Bioinformatics 2011, 6:6.12.11-6.12.19.

doi:10.1186/1471-2229-12-168

Cite this article as: Gordo et al.: High-throughput sequencing of black pepper root transcriptome. BMC Plant Biology 2012 12:168.

\section{Submit your next manuscript to BioMed Central and take full advantage of:}

- Convenient online submission

- Thorough peer review

- No space constraints or color figure charges

- Immediate publication on acceptance

- Inclusion in PubMed, CAS, Scopus and Google Scholar

- Research which is freely available for redistribution 